\title{
Evidence-Based Novel Management Options of Acute Deep Vein Thrombosis (DVT) and Prevention of DVT Recurrence in Primary Care Medicine Anno 2018 - 2020
}

\section{Jan Jacques Michiels ${ }^{1,2,3 *}$, Wim Moossdorff ${ }^{1}$, Rob Strijkers ${ }^{1}$, Mildred U Lao $^{1}$, Hans Smeets ${ }^{1}$, Ming Han ${ }^{1}$, Wilfried Schroyens ${ }^{2}$ and Alain Gadisseur $^{2}$}

${ }^{1}$ Primary Care Medicine Star-Medical Diagnostic Center, Rotterdam, the Netherlands ${ }^{2}$ Hemostasis Research Unit, Department of Hematology, University Hospital Antwerp, Antwerp Belgium

${ }^{3}$ Goodheart Institute in Nature Medicine, Blood Coagulation and Vascular Medicine Science Center, Rotterdam, the Netherlands

*Corresponding Author: Jan Jacques Michiels, Primary Care Medicine Star-Medical Diagnostic Center, Rotterdam, the Netherlands.

DOI: $10.31080 / A S M S .2020 .04 .0568$
Received: February 05, 2020

Published: February 14, 2020

(C) All rights are reserved by Jan Jacques

Michiels., et al.

\section{Abstract \\ Complete compression ultrasonography (CCUS) rules in and out acute deep vein thrombosis (DVT) and picks up alternative di- agnoses (AD) including Baker's cyst, muscle hematomas, old DVT, and superficial vein thrombosis. CCUS from the ileofemoral region to the popliteal and calf veins has become the objective test in routine daily practice to diagnose acute DVT and to classify distal, proximal and inguinal Leg Extremity Thrombosis (LET class I, II and III DVT) extension.}

Acute DVT patients are recommended to wear medical elastic stockings (MECS) for symptomatic relief of swollen legs for a few weeks. Objective testing with colour duplex ultrasonography (DUS) at time points 1, 3 and 6 months post-DVT for residual vein thrombosis is of critically importance to assess the risk for DVT recurrence and post-thrombotic syndrome (PTS) evolution. Prospective studies clearly indicate that MECS only relieves subjective symptoms of PTS but do not reduce DVT recurrence and do not improve the objective signs of PTS after long-term and lifelong follow-up.

Rapid and complete recanalization on DUS within 1 to 3 months post-DVT is associated with no reflux and low risk on DVT and PTS on the basis of which MECS and anticoagulation with vitamin K antagonist or Direct Oral anti-Xa or IIa Coagulant (DOAC) inhibitor can be withhold at 3 months post-DVT. Delayed recanalisation with residual vein thrombosis (RVT) on DUS at 3 months post-DVT is assocated with reflux due to valve destruction and a high risk of DVT recurrence and PTS indicating the need to extend anticoagulation for 6 momths to 1 year in the absence of PTS and for 2 years in the presence of PTS.

Direct Anticoagulants: DOACs preferentially apixaban twice daily have become the first line treatment option of acute DVT and PE for effective reduction of venous thromboembolism (VTE). Apixaban BID is superior to rivaroxaban OD for 3 to 6 months acute DVT/PE treatment in terms of significantly less major bleeds (MB) clinical relevant non-major (CRNM) bleeds. Low dose apixaban BID daily is the treatment of choice for extended anticoagulation in post-DVT patients at high risk of DVT recurrence. Patients with acute DVT in the ileofemoral veins are at highest risk of DVT recurrence. Catheter Directed Thrombolysis (CDT) on top of anticoagulation for the invasive treatment of acute iliofemoral deep-vein thrombosis compared with standard anticoagulant therapy (vitamine $\mathrm{K}$ antagnist or DOAC) alone is noninferior and absolutely not superior in terms of post-thrombotic syndrome sequelae after 1 year.

A prospective safety-efficacy DVT-PTS Bridging the Gap management study is proposed in patients with a first distal, proximal and iliofemoral thrombosis to reduce the overall DVT recurrence rate from about 30\% to less than 5\% patient/years to prevent PTS significantly during long-term follow-up.

Keywords: Deep Vein Thrombosis; DVT Recurrence; Posthrombotic Syndrome; D-Dimer; Compression Ultrosonography; Colour Ultrasonography; Anticoagulation; Catheter Directed Thrombolysis; Vitamin K Antagonist; Direct Oral Anticoagulants; DOAC 


\section{Introduction}

Deep vein thrombosis (DVT) as wel as alternative diagnoses (AD) such as Bakers cyst, aneurysm, hematoma, old DVT, among others mimicking DVT, are easily recognized by complete compression ultrasonography (CCUS) [1-8]. The posttest incidence of VTE after a first negative two point CUS (2-CUS) ranged from 1.7 to $3.0 \%$ indicating a negative predictive value (NPV) of 98.3 to $97 \%$ and a specificty of $98-100 \%$ for proximal DVT but overlooks distal DVT or calf vein thrombosis (CVT), indicating the need to repeat CUS within one week (Figure 1) [3,4]. Extended CUS (E-CUS) examinations covers the entire lenght of the femoral vein and the socalled subpopliteal trifurcation area (Figure 1) [4]. As E-CUS still overlooks distal vein thrombosis [5,6], Schellong., et al. introduced in 2003 complete CUS (CCUS, Figure 1) examination of all deep veins of the upper and lower leg from the groin to the ankle in a single procedure to pick up distal DVT including isolated subpopliteal and calf vein thrombosis (Figure 1) [7,8]. CCUS requires more skilled, specially trained echographist operators. For examining the proximal veins from the groin to the fossa polplitea, the patient must sit upright with legs hanging down to maximize venous filling of the calf veins. In this position CCUS can be used starting from the trifurcation of the popliteal vein to identify the posterior tibial, peroneal, gastrocnemius (medial and lateral) and the soleal (lateral and medial) veins of the lower leg. The accuracy of CCUS for the diagnosis of acute distal and proximal DVT by experienced specialists was high with a negative predictive value of $99.5 \%$ in the medical diagnostic laboratory setting or outpatient ward in three large management studies with a prevalence of DVT from $14 \%-33 \%$ [2]. The prevalences of all DVT, proximal DVT and distal DVT in 3240 patients with suspected DVT were 20.2\%, 10.0\% and $10.2 \%$ respectively [2]. Treatment of symptomatic distal calf vein thrombosis (CVT) will prevent extendion of CVT into proximal DVT thereby reducing significantly the incidence of the postthrombotic synrome (PTS) $[2,8]$.

Clinical criteria for objective grading of the postthrombotic syndrome (PTS)

The Clinical-Etiology-Anatomic-Pathophysiological (CEAP) classification was developed by phlebologists to stage chronic venous insufiency (CVI) in patients without previous thrombosis and in post-DVT patients for the classification and detection of PTS [9]. In the study by Labropoulos., et al. [11] half of the CEAP defined patients with isolated DVT (21 of 41) became asymptomatic, where- as 71 of 79 patients with CEAP defined extended DVT (EDVT, (Table 1) remained symptomatic during follow-up in the post-DVT period [10]. Reflux in the poplital/femoral region, and obstruction or outflow obstruction in the ileofemoral region or a combination of both were more common in extended proximal DVT (EDVT, Table 1) with two or more vein segments occluded as compared to isolated thrombosis with one vein segment occlusion (IDVT, Table 1) [10]. Recurrent DVT and PTS related skin damage were more likely to develop in CEAP defined multisegement EDVT than in those with isolated IDVT. Patients with combined reflux and obstruction or outflow obstruction presented with more skin damage than those with reflux or obstruction PTS alone. Distal CVT (LET class I) patients in whom leg thrombosis extended from the calf vein to the proximal poploiteal/femoral (LET class II) and/or iliofemoral veins (LET class III) produced the highest prevalence of PTS [10].
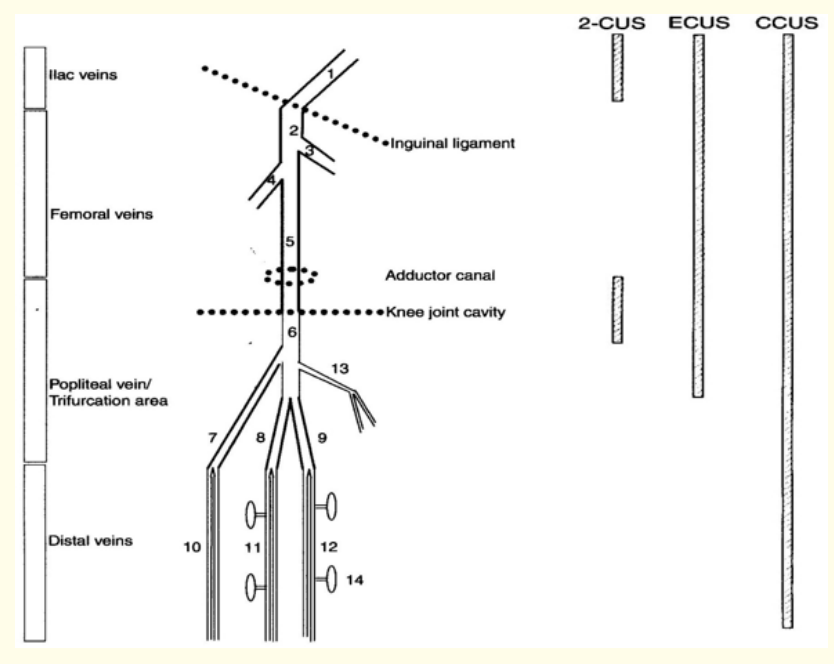

Figure 1: Schematic representation of leg veins related to wo points compression ultrasonongraphy (2-CUS) according to Lensing., et al 1989 [3], extended CUS (E-CUS) according to Cogo et al 19984, and complete CUS (CCUS) according to Schellong et al 2003 [7]. Scheme of the vein legs used since 2005 sonographists, radiologists and physicians of the Rotterdam Medical Center as fill-in form to prospectively document the location and extension of acute DVT on CCUS. Since 2012 we used the Low Extremity Thrombosis (LET) extension classification proposed by Arnoldussen and Wittens [19] as LET class I distal calf vein thrombosis, as LET class II proximal popliteal/femoral deep vein thrombosis (DVT), and as LET class III iliofemoral DVT. 
Evidence-Based Novel Management Options of Acute Deep Vein Thrombosis (DVT) and Prevention of DVT Recurrence in Primary Care Medicine Anno 2018 - 2020

\begin{tabular}{|l|c|c|c|c|c|c|}
\hline N of limbs & CEAP 0-1 & CEAP 2-3 & CEAP 4 & CEAP 5-6 & Reflux/obstruction & Recurrent DVT \\
\hline 41 IDVT \\
\hline Of which 21 symptomatic & 15 & 20 & 6 & 0 & 15 & 5 \\
\hline 79 EDVT & 10 & 40 & 21 & 8 & 61 & 16 \\
\hline Of which 71 symptomatic & 10 &
\end{tabular}

Table 1: Outcome of DVT patients with a first Isolated (IDVT) and in patients with a first Extended DVT (EDVT)[10].

Note: During a median follow-up of 3.4 years showed that half of IDVT (21 of 42 limbs) and the majority of EDVT limbs (71 of 79 limbs) become symptomatic for PTS according to the CEAP classification. Data from Labropoulos., et al [10].

Abbreviations: DVT, deep vein thrombosis; CEAP, Clinical-Etiology-Anatomic-Pathophysiological; PTS, postthrombotic syndrome.

The Rotterdam modification of Villalta score separates objective clinical (C) signs from subjective (S) symptoms and therefore becomes much more reliable for the clinically relevant evaluation of PTS at time points 6 to 12 months post-DVT. The five subjective (S) signs and seven objective clinical (C) symptoms of PTS are labeled in the literature as the Villalta score (Table 2) [11,12]. The S signs and C symptoms of the Villalta score have never been evaluated against the objective CEAP score (Table 2). The Rotterdam multidisciplinary DVT PTS working Group of primary care physicians, internists, radiologists and echographists decided to use the clinical (C) criteria of the CEAP classification for objective grading of PTS ((Table 2). The $\mathrm{C}$ of CEAP for the objective grading of leg swelling and skin lesions should be assesed at 6 months and 1 to 2 years post-DVT by physical examination by primary care physicians, internists, radiologists and surgeons. The C of CEAP in Table 2 start to appear at 6 and 12 months post-DVT and should be followed-up yearly thereafter. It is very well known that only half of the post-DVT patients with CEAP class 1 to 4 are asymptomatic $[13,14]$.

\begin{tabular}{|l|l|l|}
\hline Subjective symptoms: S of CEAP & Objective signs: C of CEAP & \\
\hline Heaviness of the foot/leg & Redness, new venous ectasia & C1 \\
Pain in calf/thigh & Varicosis & C2 \\
Cramps in calf/thigh & Edema (ankle circumference $\geq 1 \mathrm{~cm}$ ) & C3 \\
& Hyperpigmentation Induration skin & C4 \\
Pruritus Paraesthesia & Pain during calf compression (acute DVT) & C4 \\
& Ulceration of the skin & C5, C6 \\
\hline
\end{tabular}

Table 2: The Rotterdam separation of the Villalta scoring system into the subjective (S) symptoms and the objective clinical (C) of the CEAP claasification of post-thrombotic syndrome (PTS).

Separation of the Villalta scoring into subjective symptoms S of CEAP and objective clinical C of CEAP signs for assessment of PTS for the assessment at 6 months and 1 to 2 years post-DVT[2,13,14]

Abbreviations: PTS, postthrombotic syndrome; DVT, deep vein thrombosis; CEAP, Clinical-Etiology-Anatomic-Pathophysiological; C, clinical; CVI, chronic venous insufficiency; CUS, compression ultrasonography; symp, symptoms.

\section{DVT recurrence as the main cause of PTS}

Neumann and Michiels from the Phlebology Research Section of the Erasmus University Medical Center Rotterdam collaborated in scientific friendship between 2007 and 2010 and designed mulitidisciplinary prospective studies on diagnosis, prevention, and management of DVT, and prevention of DVT recurrence with ultimate aim to reduce the incidence of post-thrombotic syndrome (PTS) at the polulation level $[13,14]$ : This project resulted in the prospective Rotterdam ERASMUS DVT-PTS Bridging the Gap study design proposed by Michiels and Moossdorff on the basis of which we defined therapeutic implications to fill up some gaps between DVT and PTS related to the indication or withholding anticoagu- lation and/or wearing medical elastic stocking (MECS) (Table 3) $[13,14]$. In 2012 we introduced serial DUS at time points 1 and 3 months post-DVT for the documentation of rapid and complete versus delayed and incomplete recanalization of proximal acute DVT within 3 months post-DVT $[13,14]$. Rapid and complete recanalisation of proximal popliteal/femoral DVT (LET class II) is defined by absence of residual vein thrombosis (RVT) at time point 3 months post-DVT, which is followed by low risk of DVT recurrence and near to zero risk of reflux PTS [2]. Delayed and incomplete recanalization of proximal acute DVT in the popliteal/femoral (LET class II) is defined by residual vein thrombosis (RVT) at time point 3 months post-DVT, which has a high risk of DVT recurrence and PTS after anticoagulant discontinuation [2,13,14]. 


\begin{tabular}{|l|c|c|}
\hline LET extension & $\begin{array}{c}\text { Acute DVT on } \\
\text { CCUS }\end{array}$ & $\begin{array}{c}\text { Number/\% } \\
\text { of total }\end{array}$ \\
\hline Distal DVT: LET I & CVT & $370 / 27 \%$ \\
\hline Proximal DVT: LET II and III & CV PV FV CFV \\
or IFV & $443 / 32 \%$ \\
\hline One segment DVT & & $506 / 36 \%$ \\
\hline Iliofemoral total: LET III & & $160 / 12 \%$ \\
\hline Iliofemoral alone: LET III & & \\
\hline
\end{tabular}

Table 3: Lower Extremity Thrombosis (LET) extension of 1388 DVT legs in 1338 patients with a first acute deep vein thrombosis (DVT) on complete compression ultrasonography (CCUS)[20].

Lower Extremity Thrombosis (LET) extension: Segment 1 calf vein $(\mathrm{CV})=$ LET I; Segment 2 popliteal vein (PV) = LET II; Segment 3 femoral vein (FV) = LET II; Segment 4 common femoral vein (CFV) and segment 5 iliac vein (IFV) = LET III.

Compression stockings post-DVT do not reduce DVT recurrence and PTS

Aschwanden., et al. studied the effect of MECS to prevent PTS in a randomized clinical trial between 1997 and 2004 comparing intervention with no intervention in case of mild PTS (Figure 2) [15]. The inclusion criteria at 6 months post-DVT were early PTS with CEAP C0/1, CEAP C2, or CEAP C3 without skin lesions, and the exclusion criteria were CEAP C4 - C6 with skin lesions. The primary end point was defined as progression of mild PTS (CEAP C1 - C3) into severe PTS with skin changes (CEAP C4 or higher). The secondary end point was defined by the presence of five PTSassociated symptoms including pain, heaviness, sensation of heat, tension, and tiredness of the affected limb. The intention-to-treat population consisted of 169 post-DVT patients with early stage PTS (CEAP 1 - 3) without a skin lesion. All 169 post-DVT patients had obstruction and/or reflux PTS on DUS at 6 months post-DVT (Figure 2) [15]. The completeness of follow-up was $85 \%$ in the intervention group and $75 \%$ in the control group, with a mean follow-up of 3.2 years in the intervention group and 2.9 years in the control group. The Aschwanden study showed that wearing MECS post-DVT did not prevent the objective signs PTS progression of early CEAP 1 - 3 into advanced CEAP $\geq 4$ during long-term followup of 6 years in males as compared to control. In contrast, wearing MECS did significantly reduce event free PTS survival in females as compared to control (Figure 2). The authors concluded that recurrent DVT was a major risk factors for progression of mild PTS (CEAP 1-3) into severe PTS (C4 or higher; skin lesions) after 2 - 6 years follow-up (Figure 2) [15].
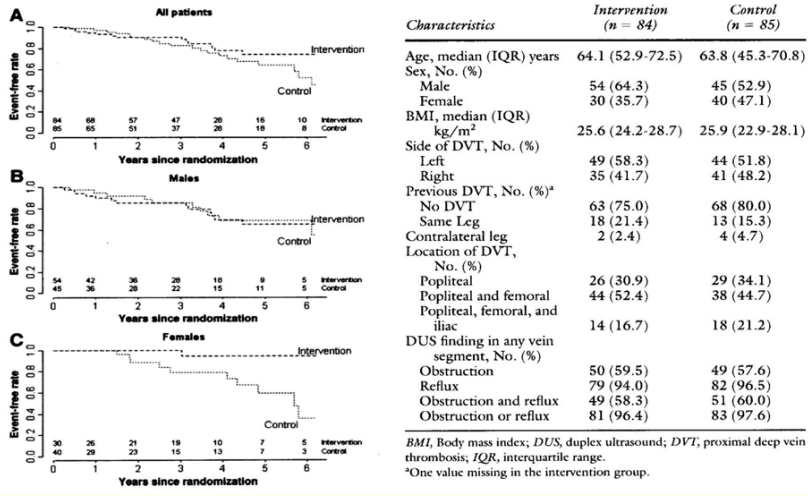

Figure 2: Randomized clinical trial in early stage PTS patients at time point 2 years post-DVT comparing intervention with MECS vs No MECS (conrols) with baseline characteristics of PTS patients who were generally symptomatic for mild PTS (CEAP 1 to $3=$ no skin lesions), no anticoagulation and abnormal DUS with reflux and/or obstruction (table right). MECS did not prevent progression of early PTS CEAP 1-3 into PTS CEAP 4 and 5 during 6 years follow-up in all and in males, but a significant reduction of PTS progression was observed in females [19].

The two prospective studies of Brandjes., et al. [16] and Pradoni., et al. [17] on the use of MECS in acute deep vein thrombosis clearly show that half of patients with a first DVT are already became completely asymptomic for PTS around time point 6 month post-DVT. This implicates that more than $50 \%$ of post-DVT patients are overtreated with MECS from 6 month to 2 years post-DVT just by the ACCP guidelines for many years all over the world. The Prandoni clinical MECS intervention study [17] and the COX epidemiological nurse study of Kahn., et al. [18] confirmed the findings of Aschwanden that MECS do not prevent the objective signs PTS [15]. DVT recurrence occurred 14 of 96 (14.6\%) in the MECS group and 13 of $98(13.3 \%)$ in the No MECS group in the study of Brandjes., et al. [16]; in 12 of 90 (13.3\%) in the MECS versus 13 of 90 (14.4\%) in the No MECS group in the study of Prandoni., et al. [17]; and in 33 of 410 (8\%) in the MECS versus 38 of 396 (9.6\%) in the No MECS group in the nurse study of Kahn., et al. [18]. The overall DVT and PE (VTE) recurrences in these 3 randomized clinical trials were 59 (10\%) versus $64(11 \%)$ in 596 post-DVT patients wearing MECS versus 396 wearing No MECS during 2 years follow-up indicating that wearing MECS doesnot prevent DVT recurrence [16-18].

Validation of the lower extremity thrombosis (LET) extension classification

Strijkers., et al. retrospectively evaluated the LET extension classification [19] using a questionaire follow-up in 1338 patients with 
Evidence-Based Novel Management Options of Acute Deep Vein Thrombosis (DVT) and Prevention of DVT Recurrence in Primary Care Medicine Anno 2018 - 2020

acute DVT collected by Dr De Maeseneer between 1991 and July 2012 at the University Hospital Antwerp [20,21]. In this Antwerp study of 1388 DVT legs in 1338 DVT patients with a first DVT on CCUS the leg vein thrombosis extension was diagnosed as distal CVT in 32\%, proximal DVT in 68\%, one segment DVT at different localizations in 32\%, ileofemoral DVT (total) in 36\% and iliofemoral DVT alone in 12\% (Table 3). Strijkers., et al. studied 315 patients responded to the questionaire by fill in forms $(23.5 \%$ of the original cohort of 1338 post-DVT patients (Table 4 and 5). The mean age in years at DVT diagnosis in 315 responders to the questionaire was $50.6 \pm 13.3$ years. DVT was left sided in $56.2 \%$. The risk factors for the first acute thrombotic event included malignan- cy (3.2\%), pregnancy/postpartum (5.7\%), immobilization/surgery (22.2\%), trauma/fracture (11.7\%), oral contraceptive use (4.8\%), not specified (21.9\%) and idiopathic (35.1\%) [21,22]. In this retrospective study PTS was scored by means of an adapted version of the original Villalta scale, a self-reported Villalta scale [12,13]. Strijkers., et al. added a visual guide to the questionaire of the clinical Villalta scale to ensure a correct assessment of the clinical situation by the patients themselves. Strijkers., et al. reasoned that there should not be a large discripancy between the original and adapted version of the Villalta scale, because the original version already incorporates a substantial patient assessed part [21,22].

\begin{tabular}{|l|c|c|c|c|c|}
\hline Clinical characteristics & Total & LET I & LET II & LET III & P-value \\
\hline Number of patients & 315 & $\mathbf{1 0 0}$ & 108 & $\mathbf{1 0 7}$ & $\mathrm{ns}$ \\
\hline Age mean, years (yrs) & 50.1 & 51.4 & 49.9 & 49.0 & $\mathrm{~ns}$ \\
\hline Follow-up median yrs & 6.9 & 6.0 & 6.3 & 7.6 & 0.005 \\
\hline Villalta median/range & $6(3-11)$ & $4(2-9)$ & $7(3-12)$ & $7(3-12)$ & 0.002 \\
\hline DVT recurrence & $29.1 \%$ & $32 \%$ & $28.7 \%$ & $27.1 \%$ & $\mathrm{~ns}$ \\
\hline Still on anticoagulation & $51.7 \%$ & $38 \%$ & $54.7 \%$ & $61.7 \%$ & 0.002 \\
\hline Still wearing MECS & $45.7 \%$ & $35 \%$ & $42.6 \%$ & $58.9 \%$ & 0.002 \\
\hline Venous ulcers & $5.4 \%$ & $6 \%$ & $2.8 \%$ & $2.8 \%$ & $\mathrm{~ns}$ \\
\hline
\end{tabular}

Table 4: Characteristics of 315 post-DVT patients after a median follow-up of 6.9 years at time of PTS Villalta score evaluation in Lower Extremity Thrombosis (LET) extension class I, versus class II and III DVT (P-value LET 1 vs II/III) [21,22].

\begin{tabular}{|c|c|c|c|c|c|}
\hline \multirow{3}{*}{$\begin{array}{l}\text { First DACUS Study } \\
\text { Number of DVT pts } \\
\text { Duration VKA }\end{array}$} & \multirow{2}{*}{\multicolumn{2}{|c|}{ No RVT on CUS }} & \multicolumn{3}{|c|}{ RVT present on CUS at 3 months } \\
\hline & & & \multicolumn{3}{|c|}{$\mathrm{N}=180$ RVT pos at 3 months } \\
\hline & 3 months & & 3 months & vs & 2 years \\
\hline Follow-up after VKA & 2 years & & 2.7 years & vs & 1 year \\
\hline Number of DVT pts & 78 & & 92 & vs & 88 \\
\hline \multicolumn{6}{|l|}{ DVT-PE recurrence: } \\
\hline In $\%$ & $1.3 \%$ & & $27.3 \%$ & vs & $19.3 \%$ \\
\hline In $\%$ per 100 pts/years & $0.63 \%$ & & $15.2 \%$ & vs & $10.0 \%$ \\
\hline Provoked / unprovoked DVT & $64 \% / 36 \%$ & & $24 \% / 76 \%$ & & \\
\hline Second DACUS Study & No RVT on CUS & & RVT prese & CUS & month \\
\hline Number of unprovoked DVT pts & 136 & vs & 258 & & \\
\hline Duration of VKA & 3 months & vs & 2 years & & \\
\hline Follow-up after VKA & 2.7 years & vs & 1 year & & \\
\hline \multicolumn{6}{|l|}{ DVT-PE recurrence } \\
\hline In $\%$ & $\mathrm{~N}=2,1.4 \%$ & vs & $\mathrm{N}=27,1$ & & \\
\hline In $\%$ per 100 pts/years & $1.4 \%$ & vs & 10.40 & & \\
\hline
\end{tabular}

Table 5: Results of prospective studies on the Duration of Anticoagulation based on Compression Ultrasonography (DACUS): the first DACUS [25] and second DACUS comparing the risk of DVT recuurence in DVT patients with rapid recanalisation (No RVT at 3 months post-DVT) versus DVT patients with delayed recanalization (RVT present on CUS at 3 months post-DVT) [25,26].

Note: the type of VTE recurrences (N=27) in the Second DACUS study was DVT only 59\%, DVT+PE 26\%, Isolated PE 14\%, and contralateral DVT 22\%. DVT = deep vein thrombosis, PE+ pulmonary embolism, VTE = venous thromboembolism. 
The role of D-dimer for DVT recurrence prevention after anticoagulant discontinuation

The 2006 PROLONG used the qualitative SIMPLIFY D-Dimer assay, which was normal in $385(63.3 \%)$ and abnormal in 223 (36.7\%) of 608 post-DVT patients (36.7\%) one month after anticoagulation discontinuation [23]. After 1.5 years follow-up the incidence of VTE recurrence increased to 24 of 385 patients with a normal Simplify D-dimer level (6.2\%); 18 events occurred among the 120 patients with increased Simplify D-dimer who stopped anticoagulation (15.0\%), as compared with 3 events among the 103 patients $(2.9 \%)$ with increased Simplify D-dimer who resumed anticoagulation [23]. After 4 years of follow-up the incidence of VTE recurrence increased to about 35\% for those with increased Simplfy DD/No VKA vs continuation of VKA; to about $20 \%$ in those with normal Simplify DD with no VKA as compared to 5.8\% with a normal Simplify DD on continuous (extended) anticoagulant for 4 years. These results after 4 years follow-up clearly indicate that separation of unprovoked DVT in two groups by a qualitative Simplify D-dimer test is feasible to separate a low vs high risk group of DVT recurrence after anticoagulant discontinuation (Palareti personal communication).

The DULCIS study (D-dimer and ultrasound in combination Italian study) for the evaluation of VTE recurrence after anticoagulation discontinuation in 988 evaluable DVT patients with a first unprovoked DVT used quantitative D-dimer assays including VIDAS, Innovance, HemosIL HS, HemosIL or STA Liatest. One months afer discontinuation the patients were followed up for 2 years [24]. In this multicenter study anticoagulation was definitely stopped in those who a normal D-dimer; 109 post-DVT patients with increased D-dimer patients refused OAT treatment. The incidence of major VTE was 8.8\% per 100 patient/years (pt/yrs) in those who refused continuation of anticoagulation; $2.8 \%$ per $100 \mathrm{pt} / \mathrm{yrs}$ in 506 post-DVT with normal D-dimer levels; and $0.7 \%$ per $100 \mathrm{pt} /$ yrs at the cost of 14 major bleedings (2.3\% pt/yrs) in 273 patients during 2 years follow-up. The DULCIS study indicate that separation of unprovoked DVT in two groups by a normal versus increasd quantitative D-dimer tests result appears to be feasible but still not sensitive enough as a test alone to separate a low versus high risk group of DVT recurrence after anticoagulant discontinuation.

\section{Prospective clinical studies on acute DVT and DVT recurrence and PTS prevention}

A prospective safety outcome management study to bridge the gap between acute DVT and subsequent PTS in patients with distal, proximal and iliofemoral vein thrombosis (CVT = LET class I, popliteofemoral DVT = LET class II and Iextending into the iliofemoral region or ilefemoral region alone = LET class III DVT) [19] is warranted with the aim to reduce the overall VTE (DVT and PE) recurrence rate from about 30\% to 3\% patient/yrs during longterm follow-up $[13,14]$. Assessment of the risk on PTS by colour duplex ultrasonography (DUS) should be performed in acute DVT patients at 1 month, 3 and 6 months, 1 year, and 2 years post-DVT to determine whether there is still a need for wearing MECS, and to see whether additional treatment is necessary $[2,13,14]$. The Maastricht LET classification can be used to standardize the documentation DVT localization and extension at time of acute DVT diagnosis. The Maastricht LET classification [19] when combined with serial CUS at 1 and 3 months post-DVT is very helpful to identify optimal treatment options in patients with acute DVT in the primary care and hospital settings (Figures 3 and 4) [2,13,14]. About one third to about half of acute DVT patients show rapid recanalization of proximal DVT with normal CUS at 3 months post-DVT (DACUS studies [25,26] and do not develop PTS after 3 - 9 months postDVT obviating the need to wear MECS and anticoagulation. In the event that reflux of the deep venous system is found at and after 3 months post-DVT, wearing MECS and extension of anticoagulation for one or a few years with vitamin $\mathrm{K}$ antagonist (VKA) or Direct Oral Anticoagulants (DOAC) are recommended (Figures 3 and 4). In the case of delayed recanalization and RVT is present at and after 3 months months post-DVT on CCUS careful prospective evaluation with regard to the need and duration of extended of anticoagulation using the Palareti's D-dimer strategy (Figure 4). Patients with symptomatic PTS C 2, 3 and 4 seems to be candidates for extended anticoagulation preferentially with DOAC in a similar low dose as used in the post-operative orthopedic surgery setting (Figures 3 and 4) $[13,14]$. Anticoagulant treatment of symptomatic distal calf vein thrombosis (LET class I) is mandatory to prevent DVT extension into the proximal popliteal and femoral veins [27-29]. Untreated calf vein thrombosis (LET class I) potentially extends into the proximal popliteal and femoral veins (LET class II) within one or a few weeks with a subsequent higher risk of VTE recurrence and PTS morbidity. About one fourth of distal vein or CVT wil extend into the popliteal and femoral region within 1 or 2 weeks (LET class II), which is associated with increased risk of DVT recurrence and PTS $[27,28]$. Extended anticoagulation is indicated in post-DVT cases with evidence reflux on DUS with or without PTS at 6 months, 12 months, and 24 months post-DVT to prevent DVT recurrence. Moossdorff and Michiels performed in 2015/2016 the DVT-PTS Bridging the Gap pilot study to test the feasability of the LET approach in the primary care setting and produced good evidence that rapid complete recanalization of proximal DVT will occur in about one third of DVT in the popliteal/femoral region (LET class II) on serial CUS within 3 months post-DVT (manuscript in preparation). A large prospective cost-effective, safety outcome study is needed whether rapid and complete recanilization at 3 months post-DVT indeed predict a low risk on DVT recurrence and that delayed and incomplete recanalization of the popliteal-femoral region 
at 3 months post-DVT (RVT present) is associated with subclinical or early PTS indicating the need to extend anticoagulation preferentially with low dose of direct oral anticoagulants (DOACs) (Figures 3 and 4).

Months(M) DUS
$\mathbf{0} \quad \mathbf{1}$
\begin{tabular}{l} 
All post DVT \\
patients (100\%) \\
Compression \\
Therapy \\
with \\
Medical Elastic \\
Compression \\
Stockings:MECS \\
\multicolumn{1}{|c|}{$\quad$ Evaluation } \\
for PTS score \\
at $1,3,6,9$ \\
and 12 months \\
\hline
\end{tabular}

\begin{tabular}{l} 
Months 1 Years \\
\hline 3
\end{tabular}

Figure 3: Prospective Rotterdam ERASMUS DVT-PTS Bridging the Gap study design proposed by Michiels and Moossdorff and therapeutic implications to bridge the gap between ATS (DVT)T and PTS related to the indication or withholding anticoagulation and/or wearing medical elastic stocking (MECS): therapeutic implications into four treatment arms at time of a first Acute Deep Vein Thrombosis (DVT) $[2,13,14]$.

Treatment arm 1. Rapid recanalization, no reflux and no PTS at 3 months post-DVT obviates the need of wearing stockings and anticoagulation at 6 months post-DVT.

Treatment arm 2. Delayed recanalization but no or mild PTS (C of CEAP: C1 C2) may indicate compensated reflux by intact calf vein pump indicating the need to extend anticoagulation for 2 years. Randomize for MECS vs No MECS at 6 months post-DVT. At 2 years post-DVT when termination of regular anticoagulant treatment may be indicated, the discontinuation of oral anticoagulation should be followed by the D-dimer strategy according to PROLONG and Dulcis studies [23,24].

Treatment arms 3 and 4 . Study arms 3 with delayed recanalization and/or, reflux on DUS and early, moderate to severe PTS (C of CEAP: C3 and C4)) at 6 and 12 months post-DVT dictates the need for extended long-term or even lifelong anticoagulation to prevent DVT recurrence as preferentially with low dose DOACs (Apixaban ${ }^{R}$ $2.5 \mathrm{mg} 2$ times per day). At time that termination of regular anticoagulant treatment may be indicated, the discontinuation of oral anticoagulation should be followed the D-dimer strategy according to the PROLONG and Dulcis studies [23,24].

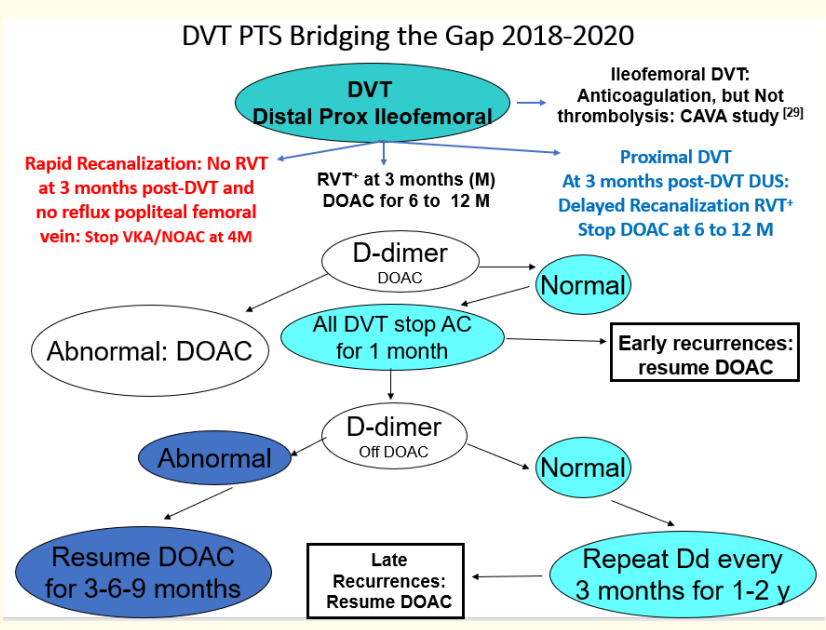

Figure 4: Algorithm propsed by Drs Michiels and Strijkers of the Rotterdam Primary Care Medical Study Group for the treatment of acute distal and proximal deep vein thrombosis (DVT), the duration of anticoagulant treatment with direct oral anticoagulants (DOACs), and the need to extend anticoagulant treatment to prevent DVT recurrence and post-DVT patients preferentially with low dose Direct Oral Anticoagulant (DOACs)[2,13,14,29, 45,46].

Acute DVT patients with isolated ileofemoral DVT and extended proximal popliteal/femoral LET class III DVT were candidate for the Maastrich randomized clinical trial comparing Catheter Directed Thrombolysis (CDT) Versus Anticoagulation since the risk of severe PTS by anticoagulation alone in LET Class III/IV ATS patients alone is irreversible and high (Figure 4) [29]. Dr Wittens of the Department of Vascular Surgery of the Maastricht University medical center performed a multicenter randomized single-blind, allocation-concelled, parallel group, supriority trial in 15 Dutch Hospitals comparing cathether directed thrombolysis and anticoagulation (CAVA study) for the prevention of postthrombotic syndrome (PTS) in patients with a first event of acute ileofemoral deep vein thrombosis [29]. Between 2010 and 2017 the CAVA study included 184 patients, who were randomly assigned to either additional ultrasoundaccelerated catheter-directed thrombolysis $(n=91)$ or standard anticoagulant treatment alone $(n=93)$. Median follow-up was $12 \cdot 0$ months (IQR $6 \cdot 0$ - 12·0). After 12-month time point follow-up, the postthromboticsyndrome occurred in $22(29 \%)$ patients allocated to additional treatment versus 26 (35\%) patients receiving standard anticoagulant treatment alone (odds ratio 0.75 [95\% CI 0.38 to 1.50$] ; \mathrm{p}=0.42$ ). Major bleeding occurred in four $(5 \%)$ patients in the intervention group, with as- 
sociated neuropraxia or the peroneal nerve in one patient, and no events in the standard treatment group. No serious adverse events occurred. None of the four deaths (one [1\%] in the interventiongroup vs three [4\%] in the standard treatment group) were treatment related. The authors concluded that additional ultrasoundaccelerated catheter-directed thrombolysis does not change the risk and sequelae of post-thrombotic syndrome 1 year after acute iliofemoral deep-vein thrombosis compared with standard anticoagulant therapy alone [29]. As the RCT design of the CAVA study is purely based on statistics and therefore inconclusive, the outcome suggests the possibility of a subgroup of acute iliefemoral DVT patients in the hands of attentive vascular surgeons who may have beneficial effect with additional ultrasound-accelerated catheterdirected thrombolysis.

Role of DOAC in the treatment of acute DVT and DVT recurrence prevention

The 2016 CHEST $10^{\text {th }}$ edition on new antithrombotic guideline for treatment of venous thromboembolism (VTE) disease recommend the Direct Oral AntiCoagulants: DOACs apixaban, rivaroxaban, edoxaban or danigatran over vitamine $\mathrm{K}$ antogonist (VKA) for the initial 3 to 6 months treatment of patients with acute DVT or pulmonary embolism (PE) [30-37]. Dabigatran inhibits thrombin (direct IIa inhibitor) and apixaban, rivaroxaban and edoxaban blocks circulating Xa (direct Xa inhibitor). The industrial driven randomized clinical trials comparing DOACs versus VKA in selected populations showed that the DOACs apixaban, rivaroxaban, and edoxaban and dabigatran are as effective as VKA therapy in the treatment of a first DVT without te need of laboratory control [30-37]. DOACs treatment of thromboembolic disease has an increased convenience for DVT and PE patients and for health care providers. In patients treated for acute DVT for 3 to 6 months, the four DOACs apixaban, rivaroxaban, LMWH-dabigatran and adjusted LMWH-edoxaban compared to vitamin K antagonist (VKA) did show equal good efficacy to prevent recurrent DVT and PE (VTE) including death from VTE between (Figure 5) [38,39]. In the treatment of acute DVT apixaban $10 \mathrm{mg}$ BID (7 days) then $5 \mathrm{mg}$ BID compared to VKA in the AMPLIFY study was superior in terms of bleeding with significantly less major bleeds or clinical relevant non-major (CRNM) bleeds compared to the other 3 DOACs with rivaroxaban 15 mg BID ( 3 weeks) then 20 mg OD, Dabigatran 150 $\mathrm{mg}$ BID and edoxaban $60 \mathrm{mg}$ OD (or adjusted $30 \mathrm{mg}$ OD) compared to VKA (Figures 5 and 6) [39,40]. Six to 18 months dabigatran extension treatment with $150 \mathrm{mg}$ BID and 12 months rivaroxaban 20 mg OD extension were associated with a significantly higher rate of any bleeding and major or clinically relevant ono-major bleeding (CRNM) as compared to placebo: hazard ration (HR) for dabigatran 3 and for rivaroxaban 5 (figure 5 bottom) [39]. In the apixaban extension study the rate of major bleeds and CRNM bleeds was $3.2 \%$ for apixaban $2.5 \mathrm{mg}$ BID and $4.3 \%$ for apixaban $5 \mathrm{mg}$ BID as compared to $2.7 \%$ in the placebo arm (HR 1 to 2,Figure 5 bottom) $[39,40]$.

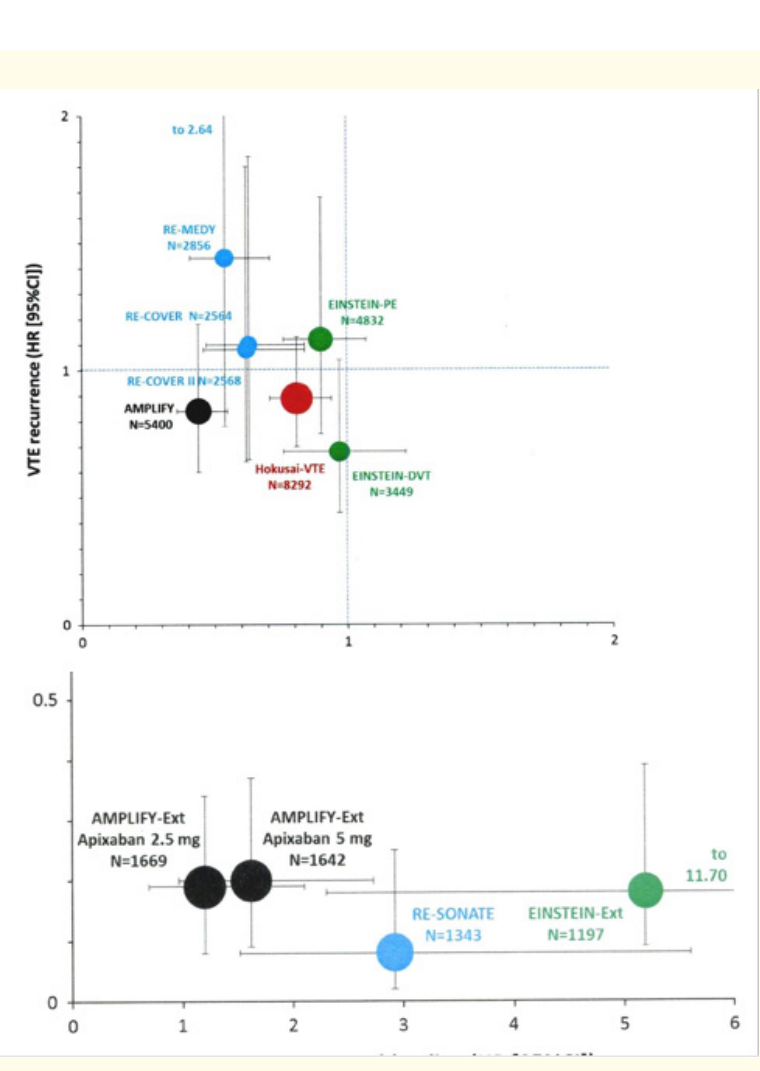

Figure 5: In total 26.872 VTE patients were included in the Amplify apixaban, Einstein DVT-PE rivaroxaban, Hokusai-VTE edoxaban and RE-COVER dabigatran studies in the efficacy meta-analysis of 3 to 6 months treatment of acute DVT or PE[30-38]. Recurrent VTE including VTE death occurred in $2.0 \%$ of 13.430 DOAC and $2.2 \%$ VKA treated patients [39]. Each of the individual DOACs showed no inferiority to VKA for VTE reduction (upper). Apixaban-VTE consistently showed superiority of less bleeding compared to VKA, whereas rivaroxaban-DVT, edoxaban-VTE and dabigatran-VTE only showed non inferiority to VKA for bleeding complications (lower). The DOACs apixaban, and rivaroxaban and LMWH-endoxaban and LMWH-dabigatran have demonstrated non-inferiority compared with standard care of VKA in the 3 to 6 months treatment of acute VTE. Apixaban BID, adjusted endoxaban OD, and dabigatran BID versus VKA were associated with significantly fewer of the combined major or clinical relevant non-majoer bleeding events (superiority), while rivaroxaban OD showed no difference versus VKA (non-inferiority) in the 3 to 6 months treatment of acute VTE (Upper figure Source Cohen., et al) $[39,40]$.

Six to 18 months apixaban and rivaroxaban treatment extension were equally superior to placebo in the prevention of recurrent VTE or death (HR 0,1 to 0,2 ). Six to 18 months dabigatran treatment extension $150 \mathrm{mg}$ BID and 12 months rivaroxaban $20 \mathrm{mg}$ OD treatment extension were associated with a significantly higher rate of any bleeding and major or clinically relevant ono-major bleeding (CRNM) as compared to placebo (HR dabigatran BID 3, rivaroxaban OD 5. In the apixaban extension study the rate of major and CRNM bleeds was 3.2\% for apixaban $2.5 \mathrm{mg}$ BID and $4.3 \%$ for apixaban $5 \mathrm{mg}$ BID as compared to $2.7 \%$ in the placebo arm (HR 1 to 2). (Lower figure, Source Cohen., et al)[39,40]. 
Results of acute VTE treatment in the meta-nalysis of Mantha and Ansell [41] comparing each of the DOACs apixaban, dabigatran,rivaroxaban and edoxaban vs VKA in terms of recurrent VTE, and details of major bleeds or CRNM bleeds are summarized clearly showed that all DOACs are non-inferior to VKA with regard to thrombotic recurrence of VTE and death (Figure 6, table 6). Apixaban is superior for less major bleeds and/or CRNM bleeds as compared to rivaroxaban, edoxaban and to a lesser extend also to dabigatran (Figure 6) [41]. Apixaban vs VKA as compared to rivaroxaban vs VKA and dabigatran vs VKA in the randomized clinal trials was also associated with less major bleeds for stroke prevention in apixaban treated patients with non-valvular atrial fibrillation [42-43]. In the Baker and Phung systemic review and adjusted indirect comparison of oral anticoagulants in atrial fibrillation comparing apixaban BID, dabigatran BID, or rivaroxaban OD or warfarin, users of apixaban BID was associated with lower risk of major, intracranial and gastrointestinal bleeding whereas rivaroxaban OD users had less associated intracranial bleeding and similar risk of major and gastrointestinal bleeding in comparison with warfarin (Figure 7) [42]. This overall evidence that apixaban BID versus rivaroxaban OD is suprior in terms of bleeding complication in all large phase III DOAC trials and meta-analysis in the two diseases VTE and AF is consistent [38-43]. Lamberts et al analysed major bleedings and persistence on oral anticoagulation (OAC) compliance in 54.321 patients with non-valvular atrial fibrillation (AF): 7973 apixaban, 6715 rivaroxaban, 15.413 dabigatran and 24.230 warfarin users (Figure 8) [44]. A total of 2418 patients (4.5\%) experienced a major bleeding event over all available follow-up for 3 years. Rivaroxaban, dabigatran, and warfarin users were significantly more likely to bleed than apixaban users with hazerd ratios of $1.49,1.17$ and 1.23 as compared to apixaban respectively [44]. Risk of nonpersistence was higher for dabigatran and warfarin initiators but similar for rivaroxaban initiators with hazard ratios of 1.45, 1.22 and 1.07 respectively as compared with apixaban initiators [44]. In this real-world cohort of nonvalvular atrial fibrillation patients, apixaban had a signifcantly lower adjusted major bleeding risk compared with rivaroxaban, dabigatran, and warfarin during a three year follow-up of DOAC treatment (Figure 8 upper) [44]. Moreover, apixaban had a lower risk of compliance loss compared with dabigatran and warfarin and similar risk of compliance loss compared with rivaroxaban during a three year follw-up period (Figure 8 lower) [44].

\begin{tabular}{|c|c|c|c|c|c|c|c|c|c|}
\hline & \multicolumn{3}{|c|}{ Dabigatran } & \multicolumn{3}{|c|}{ Rivaroxaban } & \multicolumn{3}{|c|}{ Apixaban } \\
\hline & \multicolumn{3}{|c|}{ RECOVER I and II } & \multicolumn{3}{|c|}{ EINSTEIN DVT and PE } & \multicolumn{3}{|c|}{ AMPLIFY } \\
\hline & HR & $95 \% \mathrm{CI}$ & $P$ & HR & $95 \% \mathrm{CI}$ & $\boldsymbol{P}$ & HR & $95 \% \mathrm{CI}$ & $\boldsymbol{P}$ \\
\hline Recurr & 1.09 & $0.76-1.57$ & na & 0.89 & $0.66-1.19$ & 0.41 & 0.84 & $0.60-1.18$ & $<0.001$ \\
\hline Major & 0.73 & $0.48-1.11$ & na & 0.54 & $0.37-0,79$ & 0.002 & 0.31 & $0.17-0.55$ & $<0.001$ \\
\hline Clinica & 0.62 & $0.50-0.76$ & na & 0.93 & $0.81-1.06$ & 0.27 & 0.44 & $0.36-0.55$ & $<0.001$ \\
\hline nonma & & & & & & & & & \\
\hline Death & 1.0 & $0.67-1.51$ & na & 0.89 & 0.89 & 0.43 & 0.79 & $0.53-1.19$ & na \\
\hline
\end{tabular}

Table 6: Direct Oral AntiCoagulants: DOAC treatment outcome results of Venous Thromboembolism (VTE) for 3 to 6 months compared to Vitamine K Antagonists (VKA) in terms of recurrent VTE, Major Bleeding (MB), Clinically Relevant Nonmajor Bleeding (CNMB) and Death in Randomized Clincal Trials comparing Dabigatran 150 mg BID 3 to 6 months, versus Rivaroxaban 15 mg BID three weeks then 20 mg OD 3 to 6 months, versus Apixaban 10 BID 1 week then 5 mg BID 3 to 6 months in the meta-analysis by Mantha and Ansell in the Randomized Clinical Trials RECOVER I and II (Dabigatran), EINSTEIN DVT and PE (Rivaroxaban), and AMPLIFY (Apixaban)[30-38].HR = Hazard Ratio. 95\% CI = confidential interval. P-value. BID twice (BI) daily dose. OD = Once Day dose. (na = not applicable).

Conclusions: All DOACs Dabigatran BID, Rivaroxaban OD and Apixaban BID are non-inferior to Vitamine K Antagonists (VKA) for the outcomes of VTE and Death.

Apixaban BID 3 to 6 months is significantly superior to VKA for the outcomes of Major Bleeding (MB) and Clinically Nonmajor (CNRM) Bleeding.

Rivaroxaban OD 3 to 6 months is superior to VKA for intracranial bleeding but Rivaroxaban OD 3 to 6 months is not superior for major Bleeding: MB and Clinically Relevant Nonmajor Bleeding: CNMB (Figure 6)[40].

Apixaban BID 3 to 6 months compared to Rivaroxaban OD 3 to 6 months is significantly superior for the reduction of MB and CNMB in VTE studies but also in Atrial Fibrillation studies (manuscript in preparation). 


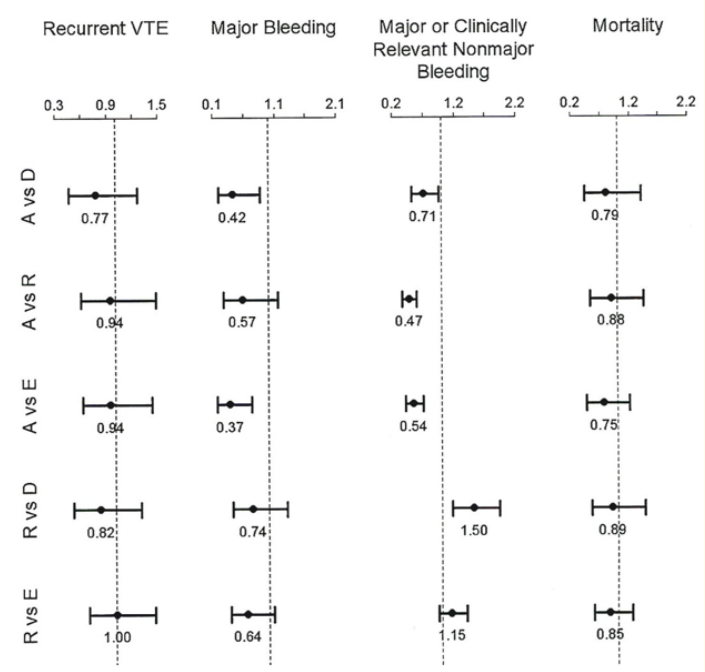

Figure 6: The estimated relative risk (RR) of recurrent VTE for apixaban BID (A) versus dabigatran BID (D); apixaban BID (A) versus rivaroxabab OD (R); and rivaroxaban OD (R) versus edoxaban OD for the treatment of acute VTE in the meta-analysis of Mantha. et al [41] demonstrate that all four DOACs are similarly effective in preventing recurrent VTE and overall survival (death) compared to standard VKA (non-inferiority). In contrast, the risk of major bleeding is significantly lower in users of apixaban BID compared with users of dabigatran BID or edoxaban OD. In addition. acute VTE treatment with apixaban BID or dabigatran BID is associated with significantly less major or clinically releant non-majoer (CRNM) (superiority) compared to rivaroxaban OD and that apixaban BID compares favorable to dabigatran BID and epoxaban OD for that endpoint [41].

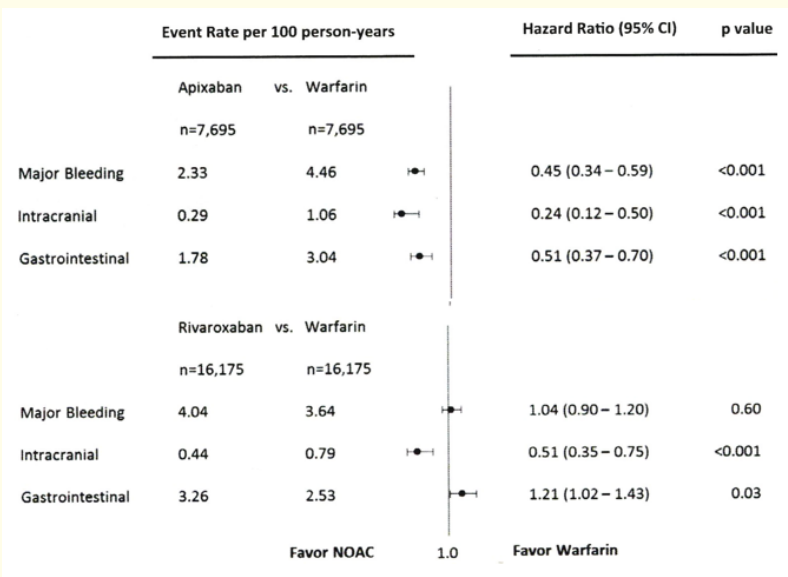

Figure 7: In the Baker and Phung systemic review and adjusted indirect comparison of oral anticoagulants in atrial fibrillation comparing apixaban BID, dabigatran BID, or rivaroxaban OD or warfarin, users of apixaban BID was associated with lower risk of major, intracranial and gastrointestinal bleeding whereas rivaroxaban OD users had less intracranial bleeding and similar risk of major and gastrointestinal bleeding in comparison with warfarin [42].

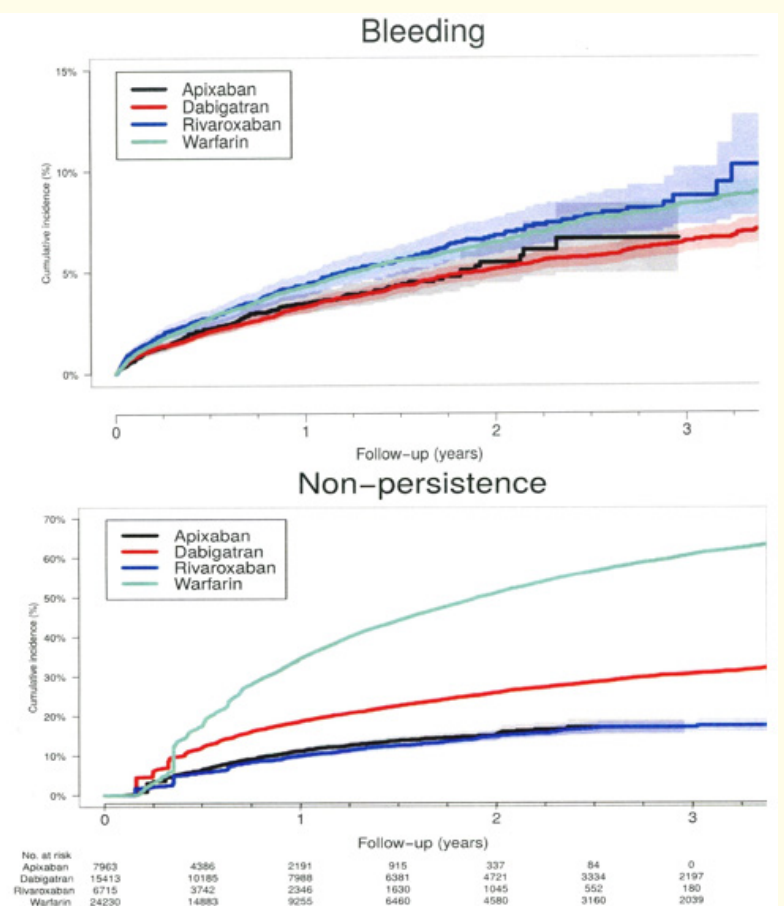

Figure 8: Lamberts et al analysed 54.321 patients with non-valvular aftrial fibrillation (AF): 7973 apixaban, 6715 rivaroxaban, 15.413 dabigatran and 24.230 warfarin users[44]. A total of 2418 patients (4.5\%) experienced a major bleeding event over all available follow-up for 3 years. Rivaroxaban OD, dabigatran BID, and warfarin users were significantly more likely to bleed than apixaban BID users with hazerd ratios of 1.49, 1.17 and 1.23 (Upper) [44]. Risk of nonpersistence was higher for dabigatran and warfarin initiators but similar for rivaroxaban initiators with hazard ratios of $1.45,1.22$ and 1.07 respectively as compared with apixaban initiators (Lower) [44].

The biological half life time of the direct Xa inhibitors apixaban, rivaroxaban and edoxaban is about 12 hours and do not differ significantly, but apixaban is administered twice daily (BID) as opposed once daily (OD) for rivaroxaban and edoxaban. Theoretically, the Xa inhibitors apixaban, rivaroxaban and edoxaban are expected equally effectivive in terms of VTE reduction and bleeding complications induction compared to VKA when dosed BID according to their pharmacokinetic and pharmacodymic characteristics. It is postulated that dosing differences of BID for apixaban versus OD for rivaroxaban and edoxaban account for the superiority of apixaban above rivaroxaban with regard to the supriority of apixaban for major bleeds and CRNM bleeds. This may be linked to higher peak levels and normal lower anti-Xa levels with OD as compared to BID to reach equal efficacy in VTE reduction at the cost of bleeding induction. This postulate has been inducted by the meta-analysis of Cohen., et al. of DOAC extension studies by the demonstration of the superiority of both apixaban $5 \mathrm{mg}$ BID and $2.5 \mathrm{mg}$ BID versus placebo in terms of major bleeds and CRNM bleeds (HR 1 to 2, Figure 
5) as compared to rivaroxaban $20 \mathrm{mg}$ OD to placebo (HR 5, Figure 5) and is in need for confirmation [39,40]. Because of the equal VTE efficacy but the significantly superior bleeding safety profile of apixaban BID compared to rivaroxaban OD, apixaban twice daily (BID) (Figure 5,Table 6) has been recomended in the UK and The Netherlands as the 'safest' first treatment option for the treatment of acute DVT, low risk PE and extended anticoagulation for prevention of DVT recurrence (Leiden University Medical Center: LUMC, VTE Study Group) with rivaroxaban edoxaban or dabigatran as a good second options according to the decision of the doctor and his fully informed VTE patient. A large quality drive randomized clinical trial directly comparing the VKA vs apixaban BID vs rivaroxaban OD in dosages as approved by the FDA and EMEA is ongoing by an independant, nonprofit organization of the PatientCentered Outcomes Researh Institute (PCORI, Dr Ortel Duke Universiy) (www.pcoci.org) in the USA This study will not solve our postulate that rivaroxaban twice daily (2x10mg BID or $2 \times 5 \mathrm{mg}$ BID) over once daily (20 mg or $10 \mathrm{OD}$ ) will be superior in terms of major bleeds and clinical relevant non-major bleeds (CRNM) risk with similar good VTE reduction in both arms of rivaroxaban BID vs OD for 12 months to prevent VTE (DVT and PE) recurrence. Large quality driven prospective Patient-Centered Outcome Research (PCOR) randomized clinical studies are warranted to directly compare the rivaroxaban BID versus the rivaroxaban OD dosing scheme in the treatment of acute DVT and for prevention DVT recurrence are warranted.

\section{Conclusion}

To bridge the gap between DVT and PTS in primary care medicine, early diagnosis of acute DVT by CCUS and anticoagulant treatment of both distal and poximal DVT is mandatory to prevent progression of distal into proximal DVT and PTS as much as possible (Figure 3 and 4) [2,13,14,45,46]. Anticoagulant treatment of symptomatic distal calf vein thrombosis (CVT) is mandatory to prevent DVT extension into proximal deep veins. CVT potentially extends into the proximal popliteal and femoral veins, which can obstruct the deep venous system and place the patient at higher risk of pulmonary embolism and more severe post-thrombotic morbidity. About one fourth of distal DVT or CVT wil extend into the popliteal and femoral region within 1 or 2 weeks, which is associated with increased risk of DVT recurrence and PTS. About one third to about half of DVT patients have normal DUS 3 months post-DVT and do not develop PTS after 3 - 9 months post-DVT obviating the need to wear MECS and anticoagulation. In the event that reflux of the deep venous system is found at 3 to 6 months post-DVT, wearing MECS and VKA or DOACs are recommended (Figures 3 and 4) $[13,14,45,46]$. At time of acute DVT isolated ileofemoral DVT and extension of proximal popliteal/femoral DVT into the ileofemoral region are candidate for Catheter Directed Thrombolysis Versus
Anticoagulation since the risk of severe PTS by anticoagulation alone in LET Class III/IV DVT patients alone is irreversible and high (Figure 4). Proximal DVTwith delayed recanalisation at 3 months post-DVT is the main cause of DVT recurrence and PTS progression. Assessment of the risk on DVT recurrence by colour duplex ultrasonography should be performed in acute DVT patients at 1 month, 3 and 6 months, 1 year, and 2 years post-DVT to determine whether there is still a need and clear indication for extended anticoagulation and wearing MECS. The LET classification of acute DVT on top of serial CUS post-DVT and D-dimer level assessment after anticoagulation discontinuation should be implemented as useful tools to better delineate the natural history and outcome of treated DVT in the multidisciplinary primary care and hospital setting $[19,45,46]$. The recent CAVA showed that additional ultrasound-accelerated catheter-directed thrombolysis on top of anticoagulation versus anticoagulation with vitamine $\mathrm{K}$ antogonist or DOAC alone does not change the risk of post-thrombotic syndrome 1 year after acute iliofemoral deep-vein thrombosis $[29,45,46]$.

\section{Declaration of Interest}

The first author Dr Michiels is the co-ordinator of the DVT-PTS Bridging the Gap project. JJ Michiels is the founder of the Goodheart Institute in Nature Medicine, Health and Diseases, Rotterdam, The Netherlands, Freedom of Science and Education, Free University Network, Europe. JJ Michiels serves as consultant professor in the Bloodcoagulation, Hemostasis Research Laboratory at the department of Hematology University Hospital, Antwerp. Writing assistance was utilised in the preparation of this manuscript, it was carried out at the Goodheart Institute and Foundation in Nature Medicine and Health, Rotterdam, The Netherlands. The authors has no other relevant affiliations or financial involvement with any organization or entity with a financial interest in or financial conflict with the subject matter or materials discussed in the manuscript apart from those disclosed. All authors of the Primary Care Medical Diagnostic Center (MDC) Rotterdam and the Bloodcaogulation Research Laboratory Antwerp collaborated with the central European Vascular Forum (CEVF) at the quality driven academic level in scientific friendship.

\section{Author Contribution}

All authors met the condidtions of substantial contributions to conception and design of the study, and the acquisition, analysis, and interpretation of data; drafting the article or revising for important intellectual content; and final approval of the version to be published.

\section{Disclosure of Conflicting Interests}

The author(s) declared no potential conflicts of interest with respect tot he research, authorship, and/or publication of this article. 


\section{Bibliography}

1. Michiels JJ., et al. "Safe exclusion of deep vein thrombosis (DVT) by a rapid sensitive ELISA D-dimer and compression ultrasonography in 1330 outpatients with suspected DVT". Angiology Ahead of Publication (2016).

2. Michiels JJ., et al. "Duplex ultrasound, clinical score, thrombotic risk, and D-dimer testing for evidence based diagnosis and management of deep vein thrombosis and alternative diagnosis in the orimary care setting and outpatient ward". International Angiology 33.1 (2014): 1-19.

3. Lensing AW., et al. "Detection of deep vein thrombosis by realtime B-mode ultrasonography". The New England Journal of Medicine 320 (1989): 342-345.

4. Cogo A., et al. "Compression ultra $\urcorner$ sonography for diagnostic management of patients with clinically suspected deep vein thrombosis: prospective cohort study". British Medical Journal 316 (1998): 17-20.

5. Birdwell BG., et al. "The clinical validity of normal compression ultrasonography in outpatients suspected of havinh deep vein thrombosis". Annals of Internal Medicine 128 (1998): 1-7.

6. Michiels JJ., et al. "Different accuracies of rapid enzyme-limked immunosorbeny, turbidimetric D-dimer asays for thrombosis exclusion: impact on diagnostic work-up of outpatients with suspected deep vein thrombosis and pulmonary embolism". Sem Thromb Hemotas 32 (2006): 687-693.

7. Schellong SM., et al. "Complete compression ultrasonography of the leg veins as a single test for the diagnosis of deep vein thrombosis". Thromb Haemost 89.2 (2003): 228-234.

8. Palareti $\mathrm{G}$ and Schellong S. "Isolated distal deep vein thrombosis: what we know and what we are doing". Journal of Thrombosis and Haemostasis 10 (2012): 11-19.

9. Eklöf B., et al. "American Venous Forum International Ad Hoc Committee for Revision of the CEAP Classification. Revision of the CEAP classification for chronic venous disorders: consensus statement". Journal of Vascular Surgery 40.6 (2004): 1248-1252.

10. Labropoulos N., et al. "The effect of venous thrombus location and the extent on the development of post-thrombotic signs and symptoms". Journal of Vascular Surgery 48.2 (2008): 407 412.

11. Prandoni P., et al. "The clinical course of deep vein thrombosis. Prospective long-term follow-up of 528 symptomatic patients". Haematologica 82 (1997): 423-428.

12. Pesavento R., et al. "Postthrombotic syndrome". Seminars in Thrombosis and Hemostasis 32.7 (2006): 744-751.
13. Michiels JJ., et al. "Prospective Studies on Diagnosis, Prevention, and Management of Deep Vein Thrombosis (DVT), DVT Recurrence and the Post-Thrombotic Syndrome (PTS): From Concept to Study Design in the Primary Care Setting". Family Medicine and Medical Science Research 3 (2014): 138.

14. Michiels JJ., et al. "Complete Compression Ultrsonography, Clinical Score, Underlying Risk Factors and D-Dimer Testing for Objective Evidence Based Diagnosis and Exclusion of Deep Vein Thrombosis and Alternative Diagnoses in the Primary Care and Hospital Setting". Journal of Hematology and Thromboembolic Diseases (2015): 3-2.

15. Aschwanden M., et al. "Effect of prolonged treatment with compression stockings to prevent post0thrombotic sequelae: a randomized controlledtrial". Journal of Vascular Surgery 47 (2008): 1015-1021.

16. Brandjes DP., et al. "Randomised trial of effect of compression stockings in patients with symptomatic proximal-vein thrombosis". Lancet 349.9054 (1997): 759-762.

17. Prandoni P., et al. "Below-knee elastic compres $\neg$ sion stockings to prevent the post-thrombotic syndrome: a randomized, controlled trial". Annals of Internal Medicine 141.4 (2004): 249256.

18. Kahn SR., et al. "SOX trial investigators. Compression stockings to prevent post-thrombotic syndrome: a ran $\neg$ domised placebo-controlled trial". Lancet 383.9920 (2014): 880-888.

19. Arnoldussen CWKP and Wittens CHA. "An imaging approach to deep vein thrombosis and the lower extremity thrombosis classification". Phlebology 27.1 (2012):143-148.

20. De Maeseneer MGR., et al. "Analysis of 1338 patients with acute lower limb deep vein thrombosis (DVT) supports the inadequacy of the term 'proximal DVT". European Journal of Vascular and Endovascular Surgery (2015).

21. Strijkers R., et al. "Validation of the LET classification. Thesis: Safety and feasibility of ultrasound accelerated cathether directed thrombolysis and the postthrombotic syndrome". Thesis 2016 Chapter 4 51-61.

22. Strijkers RHW., et al. "Proximal extension of deep vein thrombosis: does it predict postthrombotic syndrome and quality of life at long term. Thesis 2016: Safety and feasibility of ultrasound accelerated cathether directed thrombolysis and the postthrombotic syndrome". Chapter 5 63-79.

23. Palareti G., et al. "PROLONG Investigators. Simplify D-dimer testing to determine the duration of anticoagulation therapy". The New England Journal of Medicine 355 (2006): 1780-1789.

24. Palareti G., et al. "D-dimer to guide the duration of anticoagulation in patients with venous thromboembolism: a manage $\neg$ ment study". Blood 124.2 (2014):196-203. 
25. Siragusa S., et al. "Residual vein thrombosis to establish duration of anticoagulation after a first episode of deep vein thrombosis: the Duration of Anticoagulation based on Compression UltraSonography (DACUS) study". Blood 112.3 (2008): 511-515.

26. Siragusa S., et al. "Residual vein thrombosis for assessing duration of anticoagulation after unprovoked deep vein throm $\neg$ bosis of the lower limbs: the extended DACUS study". American Journal of Hematology 86.11 (2011): 914-917.

27. Lagerstet CI., et al. "Need for long-term anticoagulant treatment of symptomatic cakf vein thrombosis". Lancet 2 (1985): 515-518.

28. Palareti G. "How I teat isolated distal deep vein thrombosis (IDDVT)”. Blood 123.12 (2014): 1802-1809.

29. Notten P., et al. "Ultrasound-accelerated cathether-directed thrombolysis versus anticoagulation (CAVA) for the prevention of postthrombotic syndrome (PTS): a single-blind multicenter, randomized trial". The Lancet Haematology 7 (2020): e40-e49.

30. Agnelli G., et al. "Oral apixaban for fort he treatment of acute venous thromboembolism". The New England Journal of Medicine 369 (2013): 799-808.

31. Agnelli G., et al. "Apixaban for extended treatment of venous embolism". The New England Journal of Medicine 368 (2013): 699-708.

32. EINSTEIN Investigators., et al. "Oral rivaroxaban for symptomatic venous thromboembolism". The New England Journal of Medicine 363 (2010): 2499-2510.

33. EINSTEIN-PE Investigators., et al. "Oral rivaroxaban fort he treatment of symptomatic pulmonary emboilsm". The New England Journal of Medicine 366 (2012): 1287-1297.

34. Schulman S., et al. "Dabigatran versus warfarin in the treatment of acute venous thromboemboilsm". The New England Journal of Medicine 361 (2009): 2342-2352.

35. Schulman S., et al. "Extended use of dabigatran, warfarin or placebo in venous thromboembolism". The New England Journal of Medicine 368 (2013): 709-717.

36. Hokusai-VTE Investigators., et al. "Endoxaban versus warfarin fort he treatment of symptomatic venous thromboembolism". The New England Journal of Medicine 369 (2013): 1406-1415.

37. Schulman S., et al. "Treatment of acute venous thromboembolism with dabigatran or warfarin and pooled analysis". Circulation 129 (2014): 764-772.

38. Van Es N., et al. "Direct oral anticoagulants for acute venous thromboembolism: evidence from phase 3 trials". Blood 124 (2014): 1968-1975.
39. Cohen A., et al. "Phase III trials of new oral anticoagulants in the acute treatment and secondary prevention of VTE: comparison and critique of study methodology and results". Advances in Therapy 31 (2014): 473-493.

40. Cohen AT., et al. "Comparison of the Direct Oral Anti Coagulants (DOACs) apixaban, dabigatran and rivaroxaban in the extended treatment and prevention of VTE: systemic review and Network meta-analysis". Plos One 11.8 160-064.

41. Mantha S and Ansell J. "Indiret comparison of dabigatran, rivaroxaban, apixaban and endoxaban for the treatment of acute venous thromboembolism". Journal of Thrombosis and Thrombolysis 39 (2015): 155-165.

42. Baker WL and Phung QJ. "Systemic review and adjusted indirect comparison of oral anticoagulants in atrial fibrillation". Circulation: Cardiovascular Quality and Outcomes 5.5 (2012): 711-719.

43. Halvorson S., et al. "A nationwide registry study to compare bleeding rates in patients with atrial fibrillation being prescribed oral anticoagulation". European Heart Journal - Cardiovascular Pharmacotherapy (2017):10.109.

44. Lamberts M., et al. "Major Bleeding Complications and Persistence With Oral Anticoagulation in Non-Valvular Atrial Fibrillation: Contemporary Findings in Real-Life Danish Patients". Journal of the American Heart Association 6 (2017): e004517.

45. Michiels JJ., et al. "Diagnosis of deep vein thrombosis (DVT) and prevention of DVT recurrence and the post-thrombotic syndrome in the parimary care medicine setting anno 2014". World Journal of Critical Care Medicine 4.1 (2015): 29-39.

46. Michiels JJ., et al. "Novel evidence-based diagnosis and management og lower extremity deep vein thrombosis (DVT), prevention of DVT recurrence and the postthrombotic syndrome: personal experiences and critical appraisal of the literature 1998-2018". Family Medicine and Medical Science Research 7 (2018): 2.

\section{Assets from publication with us}

- Prompt Acknowledgement after receiving the article

- Thorough Double blinded peer review

- Rapid Publication

- Issue of Publication Certificate

- High visibility of your Published work

Website: https://www.actascientific.com/

Submit Article: https://www.actascientific.com/submission.php Email us: editor@actascientific.com

Contact us: +919182824667 